\title{
Physiological Basis of Salt Tolerance in Groundnut (Arachis hypogaea L.)
}

\author{
A. Pal" and A.K. Pal \\ Department of Plant physiology, Faculty of Agriculture, Bidhan Chandra Krishi \\ Viswavidyalaya, Mohanpur, Nadia-741252, West Bengal, India \\ *Corresponding author
}

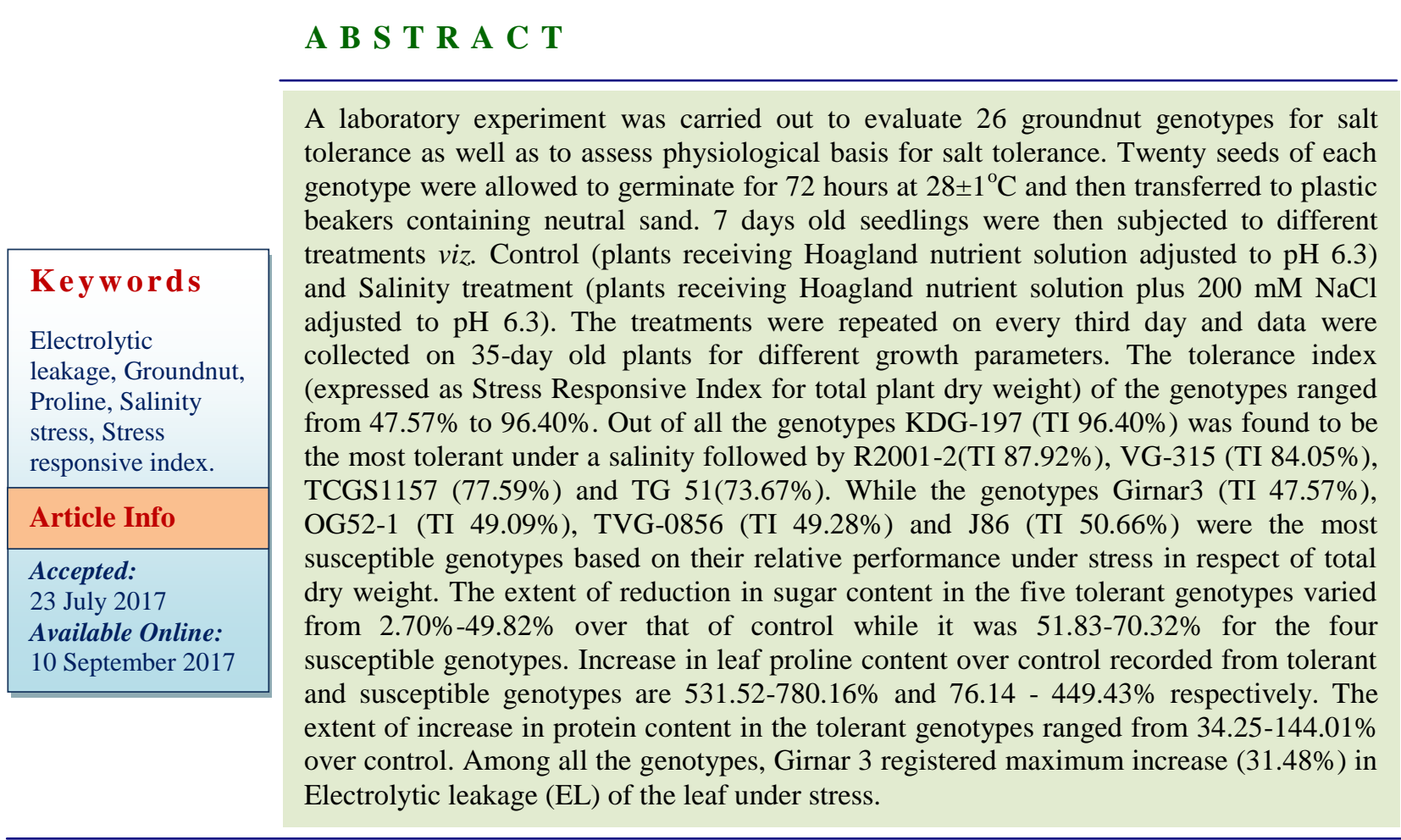

\section{Introduction}

Groundnut (Arachis hypogaea L.) is an annual legume and the 13th most important food crop and 4th most important oilseed crop of the world. In India, groundnut is an important oilseed, food and feed crop grown in an area of 6.45 million ha with a total production of 6.57 million tons based on an average of the last five years (FAO, 2005). However, different growth stages of this crop is often subjected to various types of abiotic stress like drought, salinity, high temperature etc which may cause yield loss. Soil salinity, spread in about $2.0 \mathrm{~m}$ ha of coastal and saline areas (Chhabra and Kamra, 2000) in the major groundnut growing states of India, is one of the most important abiotic factors that significantly affect seedling, vegetative and reproductive growth, seed quality and productivity. Groundnut yields have been reported to be severely affected with an 
increase in soil and water salinity (Girdhar et al 2005; Nithila et al 2013). It can affect seed germination and inhibit root and shoot growth. It interferes with water absorption leading to osmotic stress; it enhances accumulation of $\mathrm{Na}^{+}$and $\mathrm{Cl}^{-}$ions which at higher concentration may lead to cytotoxicity, impaired enzymatic function and imbalance of other elements. The cellular metabolism, biochemical as well as photosynthetic activities are all adversely affected by salt stress (Abogadallah, 2010 and Cokkizgin, 2012). One of the most effective ways to overcome salinity problems is the introduction of salt-tolerant crops. It has been reported that differences in salt tolerance exist, not only among different species, but also within certain species (Murillo-Amador et al 2001). Success of selection depends upon the amount of genetic variation present in the population. Evidence collected from various species suggests that salt tolerance is a developmentally regulated, stage-specific phenomenon, so that tolerance at one stage of development may not be correlated with tolerance at other developmental stages (Shannon, 1986).

Therefore, specific stages throughout the ontogeny of the plant, such as germination and emergence, seedling growth and its vigour, should be evaluated separately during the assessment of germplasm for salt tolerance. Such assessments may help in the development of cultivars with salt tolerance characteristics throughout the ontogeny of the plant (Bayuelo-Jimenez et al 2002).Information on salt tolerance of local groundnut varieties is scanty. The objective of the present study is therefore to screen twenty six genotypes commonly raised in Laboratory condition in sand culture to determine which genotypes can tolerate saline environments and studies on the physiological basis for salt tolerance thus help extend the present frontiers for their cultivation.

\section{Materials and Methods}

The laboratory experiment was carried out in Departmental Laboratory of Plant Physiology, Bidhan Chandra Krishi Viswavidyalaya (BCKV), Mohanpur, Nadia, West Bengal. Screening of germplasm for salinity tolerance was done with 26 genotypes of groundnut (Table 1). The seeds of all the genotypes were collected from AICRP on Groundnut, Kalyani Centre.

The seeds of 26 genotypes of groundnut were surface sterilized with $0.1 \% \mathrm{HgCl}_{2}(\mathrm{w} / \mathrm{v})$ solution for 3 minutes followed by thorough washing in distilled water. Twenty seeds were arranged in petridish of $9 \mathrm{~cm}$ diameter on Whatman No.1 filter paper and moistened with normal distilled water. The whole set were allowed to germinate for 72 hours at a temperature of $28 \pm 1{ }^{\circ} \mathrm{C}$ and relative humidity around $80 \%$. Then the germinated seeds were transferred to plastic beakers of capacity 1 liter containing neutral sand. Two replications for each genotype were made and six germinated seeds were transferred to each beaker. Full strength Hoagland solution was prepared as per modification of Epstein was supplemented to each beaker as nutrient medium. $\mathrm{pH}$ of the nutrient solution was adjusted to 6.3 each time during application of the solution. The beakers were supplemented with fresh Hoagland solution at every $3^{\text {rd }}$ day. When the seedlings were 7 days old, different treatments were initiated. The treatments were- (i) Control- plants receiving Hoagland nutrient solution adjusted to $\mathrm{pH} 6.3$ (ii) Salinity treatment- plants receiving Hoagland nutrient solution plus $200 \mathrm{mM}$ $\mathrm{NaCl}$ adjusted to $\mathrm{pH}$ 6.3. The treatments were repeated on every 3rd day. Data were collected on 35-day old plants for different growth parameters.

Growth parameters of seedlings like Length of root $(\mathrm{cm})$, Length of shoot $(\mathrm{cm})$, Total 
length of seedling $(\mathrm{cm})$, Fresh weight of root (mg), Fresh weight of shoot (mg), Fresh weight of leaf $(\mathrm{mg})$, Total fresh weight of seedling (mg), Dry weight of root (mg), Dry weight of shoot (mg), Dry weight of leaf (mg), Total dry weight of seedling (mg) and Stress response index (SRI)were measured to determine the effect of salinity treatment on seedling growth in comparison to non-stress control. Stress response index (SRI) was calculated in each case as per Chen et al (2007). It was calculated using the following formula:

Average value from stressed

SRI = Average value from control plants

For the study of physiological basis of salt tolerance some physio-biochemical analysis was also done. Extraction and estimation of total soluble sugar was done following the method of Yoshida et al (1972).The soluble protein content in the leaf was estimated following the methods of Lowry et al (1951). Proline content was determined from the germinating seed embryonic axis as per the method of Mohanty and Sridhar (1982). Electrolyte leakage was determined as described method by Guo et al (2006). Conductivity was again determined. The electrolyte leakage was calculated as the ratio of conductivity before boiling to that after boiling.

$\operatorname{EL}(\%)=\frac{(\mathrm{Cb}-\mathrm{Cw})}{-(\mathrm{Ca}-\mathrm{Cw})} \times 100$

Where,

$\mathrm{C}_{\mathrm{b}}=$ conductivity before boiling

$\mathrm{C}_{\mathrm{a}}=$ conductivity after boiling

$\mathrm{C}_{\mathrm{w}}=$ conductivity of distilled water

The experiment was set up following completely randomized design (CRD). The data were taken in triplicates and the mean data in all the cases were subjected to statistical analysis following two factor factorial design using INDOSTAT version 7.1 software. The 26 genotypes were analyzed for genetic similarity based on Euclidean distance using NTSYS-PC version 2.0 software. Dendrogram was constructed by Sequential Agglomerative Hierarical Nested (SAHN) clustering using the Un-weighted Pair Group Method with Arithmetic Mean (UPGMA) algorithm.

\section{Results and Discussion}

\section{Clustering of genotypes}

Twenty six genotypes of groundnut collected from AICRP on Groundnut, Kalyani centre, were evaluated for tolerance against $200 \mathrm{mM}$ $\mathrm{NaCl}$ salinity in sand culture using modified Hoagland solution. The mean data under stress and unstressed control condition were used for calculation of stress response index (SRI) for each genotype. Perusal of the data indicated that salinity stress reduced the length of root, shoot and total plant in all the cases except only with very few exceptions as evidenced from SRI values $<100 \%$. Out of all the genotypes, KDG-197 and TCGS1157 showed SRI of 117.89 and $123.11 \%$, respectively, for root length indicating increase in root length under stress. OG52-1 showed the highest reduction in shoot length (SRI $48.59 \%$ ) as well as whole plant length (SRI 56.26\%) registering most drastic effect of salinity stress in these two characters. Salinity stress caused reduction in fresh and dry weight of 35-day old plant as well as its different parts except for root in few genotypes. Among all the genotypes, KDG197, VG-09221 and R2001-2 recorded SRI > $100 \%$ for fresh and dry weight of root. Other three genotypes, viz., AK-343, TG-74 and VG-315 also registered SRI exceeding 100\% for root fresh weight and very high SRI for root dry weight. On the contrary, OG52-1, 
Girnar 3 and ICGV-03042 had very low mean values of SRI for both fresh and dry weight of root. The genotype KDG-197 exhibited the highest mean SRI for fresh and dry weight of leaf and total plant under stress in the present experiment. While the lowest SRI for dry weight of shoot, leaf and total plant was recorded by Girnar 3 (Table 3). The tolerance index (expressed as SRI for total plant dry weight) of the genotypes ranged from $47.57 \%$ to $96.40 \%$. Out of all the genotypes KDG-197 (TI $96.40 \%$ ) was found to be the most tolerant under a salinity stress of $200 \mathrm{mM} \mathrm{NaCl}$ and it was closely followed by R2001-2(TI 87.92\%), VG-315 (TI 84.05\%), TCGS1157 (TI 77.59\%) and TG 51(73.67\%).

While the genotypes Girnar3 (TI 47.57\%), OG52-1 (TI 49.09\%), TVG-0856 (TI 49.28\%) and J86 (TI 50.66\%) were the most susceptible genotypes based on their relative performance under stress in respect of total dry weight (Tables 2-5, Plate 1-2).

On the basis of stress response index (SRI) of root length, dry weight of root, length and fresh weight of whole plant and STIs, the twenty six genotypes were grouped following Sequential Agglomerative Hierarical Nested (SAHN) clustering on the basis of Euclidean distance. The dendrogram showed that the genotype KDG-197 separated out as a monogenotypic distinct cluster from the remaining genotypes.

Other 25 genotypes were distributed among different clusters. The four tolerant genotypes, VG-315, TCGS1157, R2001-2 and TG 51, belonged to a big cluster $\mathrm{B}$ which was separated from the other big cluster $\mathrm{A}$ at the level of coefficient value 3.36. The cluster A was further divided into two sub-clusters A1 and $\mathrm{A} 2$ at 2.87 coefficient level. The four most susceptible genotypes, J86, TVG-0856, OG52-1 and Girnar3, were distributed in different sub-clusters under A2 (Fig. 1).

\section{Physiological studies in tolerant and} susceptible genotypes

The mean data for four important biochemical parameters (viz. total soluble sugar, proline, soluble protein and electrolyte leakage) in the leaves of five tolerant (KDG-197, VG-315, TCGS1157, R2001-2 and TG 51) and four susceptible genotypes (J86, TVG-0856, OG52-1 and Girnar 3) have been presented in table 6. Perusal of the data indicated that all the nine genotypes showed reduction in leaf sugar content under salinity stress. The five tolerant genotypes registered $2.70 \%$ to $49.82 \%$ reduction in sugar content over that of control whereas the extent of reduction in the four susceptible genotypes varied from $51.83-70.32 \%$ over control. Salinity stress in the present experiment caused remarkable increase in leaf proline content in the selected genotypes under study except in Girnar 3 which only registered $76.14 \%$ increase over control. In the present experiment, the tolerant genotypes registered 531.52-780.16\% increase in proline content over control with R2001-2 showing the highest increase. On the contrary, the susceptible genotypes recorded $76.14-449.43 \%$ increase over control in the leaf proline content. All the nine genotypes recorded increase in leaf soluble protein content under $200 \mathrm{mM} \mathrm{NaCl}$ treatment. The susceptible genotypes exhibited 1.58-42.58\% increase in leaf protein content with the most susceptible genotype (Girnar 3) registering the minimum increase over control. The extent of increase in protein content in the tolerant genotypes ranged from 34.25$144.01 \%$ over control. Among all the genotypes, KDG-197 registered then maximum increase in soluble content under salinity stress and it was also the most tolerant genotype identified in the present experiment. The genotypes under study exhibited increase in electrolyte leakage (EL) of the leaf when exposed to salinity treatment. Among all the genotypes, Girnar 3 registered maximum 
increase $(31.48 \%)$ in EL of the leaf under stress and it might be noted this genotypes was the most susceptible among all. Two genotypes, KDG-197 (6.48\%) and R2001-2
$(3.00 \%)$ registered very small increase in EL\% in comparison with the unstressed control and ranked at the top among the tolerant genotypes.

Plate.1 Effect of salinity stress on seedling growth of Tolerant genotypes of groundnut
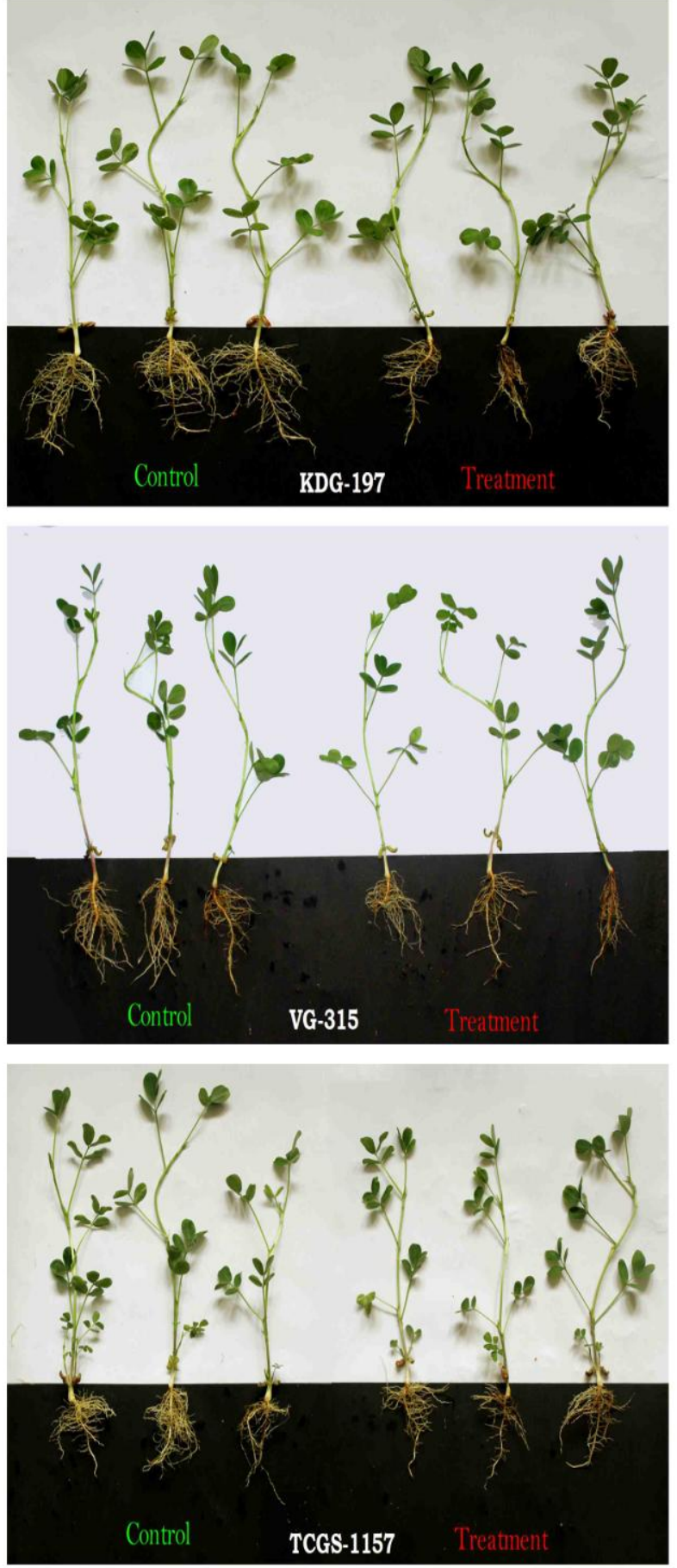
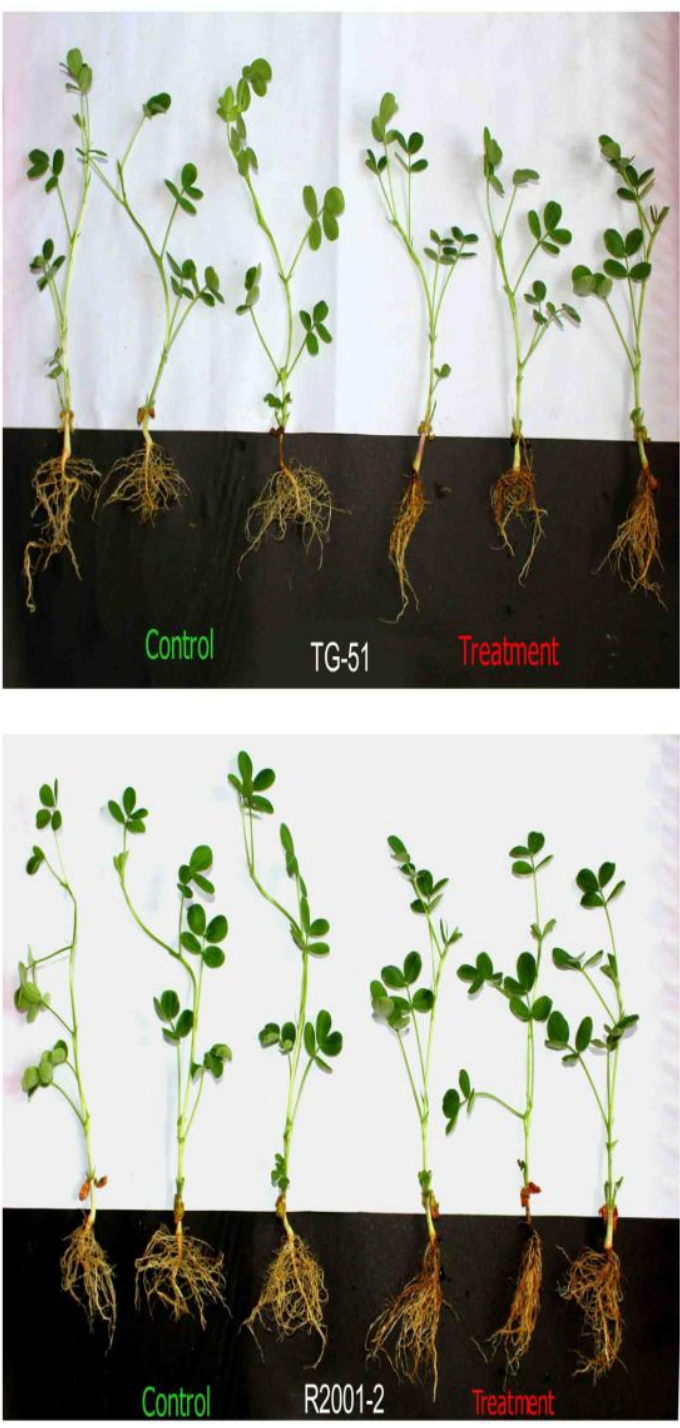
Plate.2 Effect of salinity stress on seedling growth of susceptible genotypes of groundnut
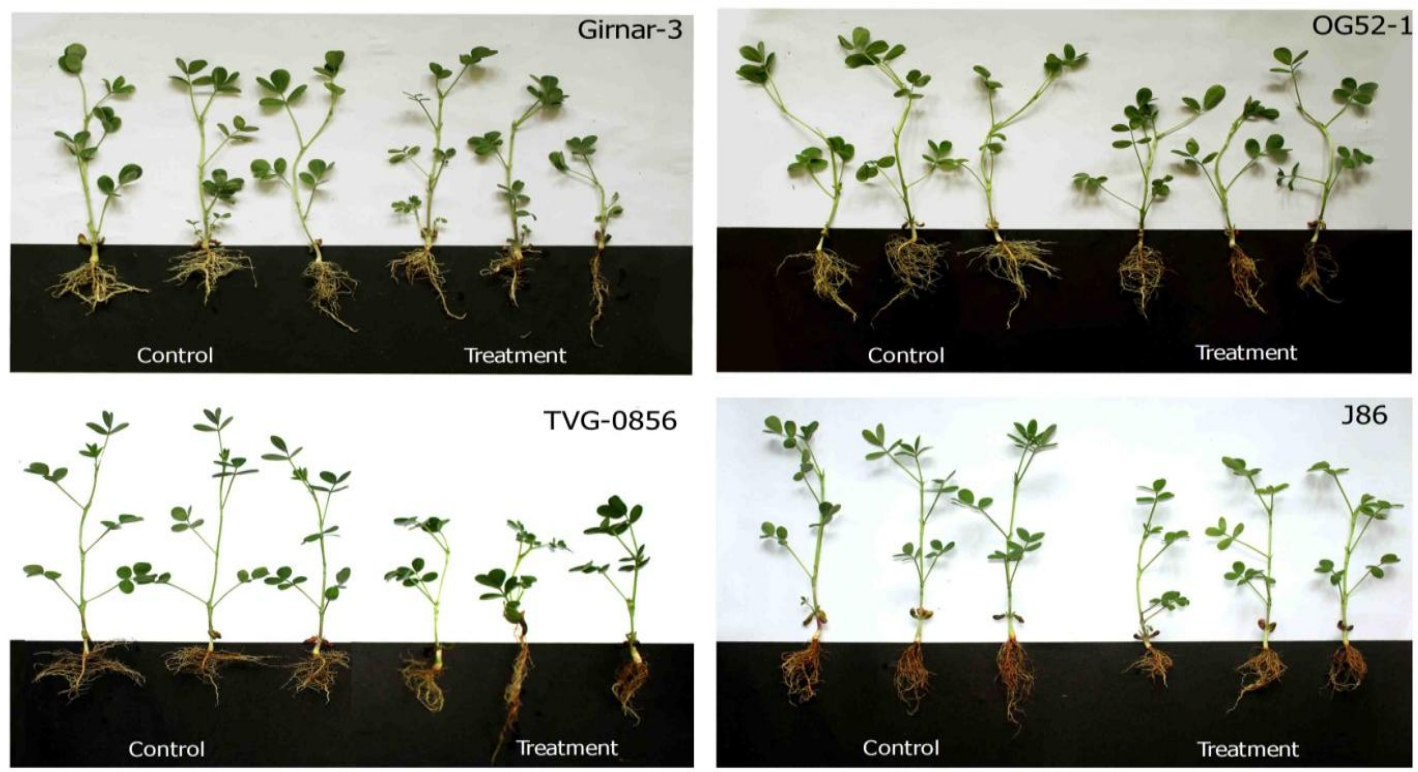

Table.1 List of genotypes used in the experiment

\begin{tabular}{|c|c|c|c|c|c|}
\hline Sl. No. & Genotype & Sl. No. & Genotype & Sl. No. & Genotype \\
\hline 1 & AK-335 & 10 & VG-09221 & 19 & TG-75 \\
\hline 2 & KDG-197 & 11 & ICGV-07038 & 20 & ICGV-03042 \\
\hline 3 & ICGV-05155 & 12 & ICGV-06138 & 21 & OG52-1 \\
\hline 4 & AK-343 & 13 & TG-74 & 22 & Girnar3 \\
\hline 5 & ICGV-03043 & 14 & TVG-0856 & 23 & CGMG-2010 \\
\hline 6 & Dh235 & 15 & JCG-3005 & 24 & R2001-2 \\
\hline 7 & ICGV-06420 & 16 & LGN163 & 25 & CTMG11 \\
\hline 8 & J86 & 17 & VG-315 & 26 & TG-51 \\
\hline 9 & JCG-2141 & 18 & TCGS1157 & & \\
\hline
\end{tabular}


Table.2 Effect of salinity stress on length of root, shoot and whole plant of 26 genotypes of groundnut

\begin{tabular}{|c|c|c|c|c|c|c|c|}
\hline \multirow{2}{*}{\multicolumn{2}{|c|}{ Genotype }} & \multicolumn{2}{|c|}{ Root length $(\mathrm{cm})$} & \multicolumn{2}{|c|}{ Shoot length $(\mathrm{cm})$} & \multicolumn{2}{|c|}{ Total length $(\mathrm{cm})$} \\
\hline & & Control & Treatment & Control & Treatment & Control & Treatment \\
\hline 1 & AK-335 & 4.80 & $4.37(-9.09)$ & 8.79 & $6.71(-23.63)$ & 13.59 & $11.08(-18.49)$ \\
\hline 2 & KDG-197 & 5.48 & $6.46(+17.89)$ & 11.59 & $9.30(-19.76)$ & 17.07 & $15.76(-7.68)$ \\
\hline 3 & ICGV-05155 & 6.12 & $5.56(-9.20)$ & 10.50 & $7.74(-26.32)$ & 16.62 & $13.29(-20.06)$ \\
\hline 4 & AK-343 & 5.30 & $4.40(-17.03)$ & 8.61 & $6.74(-21.68)$ & 13.91 & $11.14(-19.89)$ \\
\hline 5 & ICGV-03043 & 5.82 & $5.60(-3.83)$ & 9.23 & $6.09(-34.08)$ & 15.06 & $11.69(-22.38)$ \\
\hline 6 & Dh235 & 5.21 & $4.67(-10.37)$ & 8.53 & $6.69(-21.60)$ & 13.74 & $11.36(-17.32)$ \\
\hline 7 & ICGV-06420 & 4.67 & $3.12(-33.26)$ & 7.04 & $5.22(-25.81)$ & 11.71 & $8.34(-28.83)$ \\
\hline 8 & J86 & 5.63 & $4.37(-22.48)$ & 12.00 & $8.10(-32.50)$ & 17.63 & $12.47(-29.30)$ \\
\hline 9 & JCG-2141 & 6.70 & $5.01(-25.22)$ & 8.07 & $6.50(-19.42)$ & 14.77 & $11.51(-22.03)$ \\
\hline 10 & VG-09221 & 6.80 & $4.59(-32.50)$ & 8.91 & $7.16(-19.68)$ & 15.71 & $11.75(-25.24)$ \\
\hline 11 & ICGV-07038 & 6.04 & $4.20(-30.50)$ & 8.72 & $6.41(-26.46)$ & 14.76 & $10.61(-28.13)$ \\
\hline 12 & ICGV-06138 & 5.72 & $4.74(-17.08)$ & 9.16 & $6.27(-31.51)$ & 14.88 & $11.02(-25.96)$ \\
\hline 13 & TG-74 & 3.73 & $3.04(-18.41)$ & 10.03 & $5.08(-49.37)$ & 13.76 & $8.12(-40.95)$ \\
\hline 14 & TVG-0856 & 4.05 & $2.93(-27.63)$ & 7.06 & $4.80(-31.98)$ & 11.11 & $7.73(-30.39)$ \\
\hline 15 & JCG-3005 & 6.34 & $4.41(-30.41)$ & 9.40 & $6.70(-28.72)$ & 15.74 & $11.11(-29.42)$ \\
\hline 16 & LGN163 & 5.57 & $4.67(-16.17)$ & 11.41 & $7.62(-33.19)$ & 16.98 & $12.29(-27.61)$ \\
\hline 17 & VG-315 & 4.63 & $3.90(-15.83)$ & 9.93 & $9.09(-8.49)$ & 14.57 & $12.99(-10.82)$ \\
\hline 18 & TCGS1157 & 5.52 & $6.80(+23.25)$ & 8.92 & $6.00(-32.72)$ & 14.45 & $12.80(-11.42)$ \\
\hline 19 & TG-75 & 5.32 & $4.33(-18.55)$ & 8.56 & $5.36(-37.45)$ & 13.89 & $9.69(-30.22)$ \\
\hline 20 & ICGV-03042 & 4.32 & $3.75(-13.34)$ & 9.80 & $7.08(-27.76)$ & 14.12 & $10.82(-23.39)$ \\
\hline 21 & OG52-1 & 6.13 & $4.18(-31.90)$ & 9.46 & $4.60(-51.39)$ & 15.60 & $8.78(-43.74)$ \\
\hline 22 & Girnar3 & 3.32 & $2.37(-28.69)$ & 6.75 & $4.32(-36.03)$ & 10.08 & $6.69(-33.61)$ \\
\hline 23 & CGMG-2010 & 4.42 & $4.34(-1.74)$ & 10.11 & $7.75(-23.31)$ & 14.53 & $12.10(-16.75)$ \\
\hline 24 & R2001-2 & 5.87 & $5.72(-2.50)$ & 9.69 & $6.98(-28.00)$ & 15.56 & $12.70(-18.38)$ \\
\hline 25 & CTMG11 & 6.08 & $5.51(-9.33)$ & 8.74 & $5.62(-35.68)$ & 14.82 & $11.13(-24.90)$ \\
\hline 26 & TG-51 & 5.59 & $5.18(-7.39)$ & 8.10 & $6.29(-22.35)$ & 13.69 & $11.47(-16.24)$ \\
\hline & & S.E. $\mathrm{m}( \pm)$ & C.D. $5 \%$ & S.E. $\mathrm{m}( \pm)$ & C.D. $5 \%$ & S.E. $\mathrm{m}( \pm)$ & C.D. $5 \%$ \\
\hline & Genotype(G) & 0.07 & 0.14 & 0.11 & 0.22 & 0.14 & 0.28 \\
\hline & Treatment(T) & 0.02 & 0.04 & 0.03 & 0.06 & 0.04 & 0.08 \\
\hline & $\mathrm{G} \times \mathrm{T}$ & 0.10 & 0.20 & 0.16 & 0.31 & 0.20 & 0.40 \\
\hline
\end{tabular}

Means showing the same letters in a column do not differ significantly at a 5\% probability level.

Data in parentheses indicate percentage increase (+) or decrease (-) over control 
Table.3 Effect of salinity stress on fresh weight of 35-day old plant and its different parts in 26 genotype of groundnut

\begin{tabular}{|c|c|c|c|c|c|c|c|c|c|}
\hline \multirow{2}{*}{\multicolumn{2}{|c|}{ Genotype }} & \multicolumn{2}{|c|}{ Root Fresh Weight(g) } & \multicolumn{2}{|c|}{ Shoot Fresh Weight(g) } & \multicolumn{2}{|c|}{ Leaf Fresh Weight(g) } & \multicolumn{2}{|c|}{ Total Fresh Weight $(\mathrm{g})$} \\
\hline & & Control & Treatment & Control & Treatment & Control & Treatment & Control & Treatment \\
\hline 1 & AK-335 & 1.20 & $0.89(-25.9)$ & 2.32 & $1.25(-46.20)$ & 1.90 & $0.77(-59.23)$ & 5.42 & $2.91(-46.28)$ \\
\hline 2 & KDG-197 & 2.02 & $2.09(3.47)$ & 2.98 & $2.87(-3.69)$ & 2.34 & $1.84(-21.48)$ & 7.35 & $6.80(-7.44)$ \\
\hline 3 & ICGV-05155 & 1.39 & $0.89(-36.21)$ & 2.17 & $1.99(-8.43)$ & 1.78 & $1.23(-30.96)$ & 5.34 & $4.10(-23.16)$ \\
\hline 4 & AK-343 & 1.18 & $1.22(3.11)$ & 2.29 & $1.48(-35.13)$ & 1.87 & $0.91(-51.16)$ & 5.34 & $3.61(-32.29)$ \\
\hline 5 & ICGV-03043 & 0.95 & $0.72(-24.47)$ & 1.93 & $1.17(-39.31)$ & 1.58 & $0.73(-54.01)$ & 4.47 & $2.62(-41.39)$ \\
\hline 6 & Dh235 & 1.90 & $1.56(-17.72)$ & 2.91 & $1.98(-31.85)$ & 2.38 & $1.23(-48.46)$ & 7.19 & $4.78(-33.52)$ \\
\hline 7 & ICGV-06420 & 1.50 & $1.23(-17.78)$ & 2.31 & $1.40(-39.31)$ & 1.89 & $0.87(-54.06)$ & 5.69 & $3.50(-38.52)$ \\
\hline 8 & J86 & 1.64 & $1.63(-0.81)$ & 2.16 & $1.66(-23.00)$ & 1.77 & $1.03(-41.70)$ & 5.57 & $4.32(-22.40)$ \\
\hline 9 & JCG-2141 & 1.45 & $0.32(-77.70)$ & 2.63 & $1.76(-32.95)$ & 2.15 & $1.09(-49.38)$ & 6.23 & $3.18(-49.01)$ \\
\hline 10 & VG-09221 & 1.21 & $1.58(30.58)$ & 2.25 & $1.75(-22.34)$ & 1.84 & $1.08(-41.13)$ & 5.30 & $4.41(-16.73)$ \\
\hline 11 & ICGV-07038 & 1.24 & $1.18(-5.09)$ & 2.47 & $1.30(-47.43)$ & 2.02 & $0.80(-60.40)$ & 5.72 & $3.28(-42.69)$ \\
\hline 12 & ICGV-06138 & 1.16 & $0.70(-39.77)$ & 2.21 & $1.15(-48.11)$ & 1.81 & $0.71(-60.70)$ & 5.17 & $2.56(-50.58)$ \\
\hline 13 & TG-74 & 0.96 & $1.20(25.34)$ & 1.54 & $1.23(-19.96)$ & 1.26 & $0.76(-39.26)$ & 3.76 & $3.20(-14.91)$ \\
\hline 14 & TVG-0856 & 1.20 & $0.83(-30.92)$ & 2.54 & $1.23(-51.64)$ & 2.08 & $0.76(-63.30)$ & 5.82 & $2.82(-51.58)$ \\
\hline 15 & JCG-3005 & 1.31 & $1.06(-19.34)$ & 2.26 & $2.06(-9.00)$ & 1.85 & $1.27(-31.35)$ & 5.42 & $4.38(-19.13)$ \\
\hline 16 & LGN163 & 1.34 & $0.81(-39.65)$ & 1.72 & $1.15(-32.95)$ & 1.41 & $0.71(-49.29)$ & 4.46 & $2.67(-40.06)$ \\
\hline 17 & VG-315 & 1.44 & $1.74(20.88)$ & 2.59 & $2.38(-8.23)$ & 2.12 & $1.47(-30.66)$ & 6.15 & $5.59(-9.10)$ \\
\hline 18 & TCGS1157 & 1.81 & $1.76(-2.76)$ & 2.84 & $2.07(-27.00)$ & 2.32 & $1.28(-44.76)$ & 6.98 & $5.12(-26.60)$ \\
\hline 19 & TG-75 & 1.31 & $1.06(-19.13)$ & 2.26 & $1.44(-36.52)$ & 1.85 & $0.89(-51.89)$ & 5.42 & $3.38(-37.58)$ \\
\hline 20 & ICGV-03042 & 2.32 & $0.91(-60.78)$ & 2.14 & $1.26(-41.19)$ & 1.74 & $0.78(-55.26)$ & 6.20 & $2.94(-52.50)$ \\
\hline 21 & OG52-1 & 1.83 & $1.08(-40.88)$ & 2.63 & $1.54(-41.37)$ & 2.15 & $0.95(-55.59)$ & 6.60 & $3.57(-45.83)$ \\
\hline 22 & Girnar3 & 1.99 & $1.07(-46.40)$ & 2.59 & $1.49(-42.47)$ & 2.12 & $0.92(-56.60)$ & 6.70 & $3.48(-48.08)$ \\
\hline 23 & CGMG-2010 & 1.08 & $1.18(8.95)$ & 1.87 & $1.78(-4.64)$ & 1.53 & $1.10(-27.73)$ & 4.48 & $4.06(-9.23)$ \\
\hline 24 & R2001-2 & 1.49 & $1.56(4.25)$ & 2.65 & $2.16(-18.26)$ & 2.17 & $1.34(-38.31)$ & 6.31 & $5.06(-19.77)$ \\
\hline 25 & CTMG11 & 1.85 & $1.14(-38.31)$ & 2.47 & $1.33(-46.29)$ & 2.02 & $0.82(-59.41)$ & 6.34 & $3.29(-48.03)$ \\
\hline 26 & TG-51 & 1.64 & $1.21(-26.42)$ & 1.97 & $1.94(1.36)$ & 1.61 & $1.20(-25.26)$ & 5.22 & $4.35(-16.60)$ \\
\hline & & S.E. $m( \pm)$ & C.D. $5 \%$ & S.E. $m( \pm)$ & C.D. $5 \%$ & S.E. $\mathrm{m}( \pm)$ & C.D. $5 \%$ & S.E. $m( \pm)$ & C.D. $5 \%$ \\
\hline & Genotype $(\mathrm{G})$ & 0.02 & 0.04 & 0.03 & 0.05 & 0.14 & 0.28 & 0.15 & 0.29 \\
\hline & Treatment(T) & 0.01 & 0.01 & 0.01 & 0.01 & 0.04 & 0.08 & 0.04 & 0.08 \\
\hline & $\mathrm{G} \times \mathrm{T}$ & 0.03 & 0.05 & 0.04 & 0.08 & 0.20 & 0.39 & 0.21 & 0.42 \\
\hline
\end{tabular}

Means showing the same letters in a column do not differ significantly at a 5\% probability level.

Data in parentheses indicate percentage increase $(+)$ or decrease $(-)$ over control 
Table.4 Effect of salinity stress on fresh weight of 35-day old plant and its different parts in 26 genotype of groundnut

\begin{tabular}{|c|c|c|c|c|c|c|c|c|c|}
\hline \multirow{2}{*}{\multicolumn{2}{|c|}{ Genotype }} & \multicolumn{2}{|c|}{ Root dry weight(g) } & \multicolumn{2}{|c|}{ Shoot dry weight(g) } & \multicolumn{2}{|c|}{ Leaf dry weight(g) } & \multicolumn{2}{|c|}{ Total dry weight(g) } \\
\hline & & Control & Treatment & Control & Treatment & Control & Treatment & Control & Treatment \\
\hline 1 & AK-335 & 0.10 & $0.09(-12.88)$ & 0.34 & $0.21(-38.83)$ & 0.20 & $0.09(-54.11)$ & 0.65 & $0.39(-39.49)$ \\
\hline 2 & KDG-197 & 0.16 & $0.21(+33.31)$ & 0.61 & $0.58(-4.40)$ & 0.34 & $0.28(-19.40)$ & 1.11 & $1.07(-3.89)$ \\
\hline 3 & ICGV-05155 & 0.10 & $0.06(-40.00)$ & 0.33 & $0.23(-29.30)$ & 0.19 & $0.10(-45.63)$ & 0.62 & $0.40(-35.48)$ \\
\hline 4 & AK-343 & 0.13 & $0.12(-5.15)$ & 0.44 & $0.31(-28.26)$ & 0.26 & $0.14(-45.46)$ & 0.83 & $0.58(-30.52)$ \\
\hline 5 & ICGV-03043 & 0.09 & $0.07(-28.51)$ & 0.37 & $0.20(-46.36)$ & 0.21 & $0.09(-57.81)$ & 0.68 & $0.35(-49.01)$ \\
\hline 6 & Dh235 & 0.12 & $0.08(-33.33)$ & 0.41 & $0.23(-43.55)$ & 0.25 & $0.11(-56.75)$ & 0.78 & $0.42(-45.50)$ \\
\hline 7 & ICGV-06420 & 0.15 & $0.15(-0.00)$ & 0.39 & $0.22(-42.25)$ & 0.23 & $0.10(-55.89)$ & 0.76 & $0.47(-38.43)$ \\
\hline 8 & J86 & 0.20 & $0.13(-35.00)$ & 0.51 & $0.26(-48.69)$ & 0.30 & $0.12(-59.56)$ & 1.00 & $0.51(-49.17)$ \\
\hline 9 & JCG-2141 & 0.20 & $0.15(-25.00)$ & 0.46 & $0.26(-44.59)$ & 0.27 & $0.12(-57.30)$ & 0.94 & $0.52(-44.33)$ \\
\hline 10 & VG-09221 & 0.10 & $0.10(-0.00)$ & 0.41 & $0.27(-33.87)$ & 0.24 & $0.12(-49.32)$ & 0.75 & $0.50(-33.33)$ \\
\hline 11 & ICGV-07038 & 0.10 & $0.11(+13.75)$ & 0.36 & $0.23(-35.52)$ & 0.21 & $0.10(-51.57)$ & 0.66 & $0.44(-34.16)$ \\
\hline 12 & ICGV-06138 & 0.12 & $0.08(-33.33)$ & 0.29 & $0.19(-34.48)$ & 0.15 & $0.09(-40.00)$ & 0.56 & $0.36(-35.93)$ \\
\hline 13 & TG-74 & 0.09 & $0.08(-11.11)$ & 0.25 & $0.19(-23.69)$ & 0.15 & $0.09(-40.00)$ & 0.49 & $0.36(-26.35)$ \\
\hline 14 & TVG-0856 & 0.13 & $0.08(-39.46)$ & 0.35 & $0.18(-48.12)$ & 0.21 & $0.08(-61.90)$ & 0.69 & $0.34(-51.20)$ \\
\hline 15 & JCG-3005 & 0.24 & $0.08(-66.67)$ & 0.48 & $0.37(-22.23)$ & 0.28 & $0.17(-41.16)$ & 1.00 & $0.62(-38.00)$ \\
\hline 16 & LGN163 & 0.13 & $0.06(-52.64)$ & 0.24 & $0.16(-34.24)$ & 0.14 & $0.07(-50.00)$ & 0.51 & $0.30(-41.82)$ \\
\hline 17 & VG-315 & 0.13 & $0.12(-5.29)$ & 0.60 & $0.55(-8.83)$ & 0.36 & $0.25(-30.84)$ & 1.09 & $0.91(-15.96)$ \\
\hline 18 & TCGS1157 & 0.17 & $0.13(-23.53)$ & 0.50 & $0.43(-14.66)$ & 0.30 & $0.19(-35.96)$ & 0.97 & $0.75(-22.42)$ \\
\hline 19 & TG-75 & 0.17 & $0.11(-37.24)$ & 0.47 & $0.33(-30.00)$ & 0.27 & $0.14(-47.57)$ & 0.91 & $0.58(-36.40)$ \\
\hline 20 & ICGV-03042 & 0.17 & $0.06(-64.01)$ & 0.28 & $0.22(-21.43)$ & 0.16 & $0.10(-36.74)$ & 0.61 & $0.38(-37.50)$ \\
\hline 21 & OG52-1 & 0.19 & $0.09(-53.56)$ & 0.46 & $0.25(-44.93)$ & 0.27 & $0.11(-59.26)$ & 0.92 & $0.45(-50.91)$ \\
\hline 22 & Girnar3 & 0.16 & $0.10(-40.78)$ & 0.46 & $0.23(-50.36)$ & 0.27 & $0.10(-62.96)$ & 0.89 & $0.43(-52.06)$ \\
\hline 23 & CGMG-2010 & 0.13 & $0.10(-23.08)$ & 0.39 & $0.30(-24.56)$ & 0.23 & $0.13(-42.86)$ & 0.76 & $0.53(-29.96)$ \\
\hline 24 & R2001-2 & 0.11 & $0.10(-6.09)$ & 0.44 & $0.41(-5.36)$ & 0.26 & $0.18(-28.59)$ & 0.80 & $0.70(-12.45)$ \\
\hline 25 & CTMG11 & 0.17 & $0.09(-45.12)$ & 0.49 & $0.34(-30.82)$ & 0.29 & $0.15(-47.68)$ & 0.94 & $0.58(-38.16)$ \\
\hline 26 & TG-51 & 0.15 & $0.10(-31.13)$ & 0.61 & $0.50(-17.94)$ & 0.36 & $0.23(-37.60)$ & 1.12 & $0.83(-26.40)$ \\
\hline & & S.E. $m( \pm)$ & C.D. $5 \%$ & S.E. $\mathrm{m}( \pm)$ & C.D. $5 \%$ & S.E. $\mathrm{m}( \pm)$ & C.D. $5 \%$ & S.E. $m( \pm)$ & C.D. $5 \%$ \\
\hline & Genotype(G) & 0.017 & 0.04 & 0.05 & 0.10 & 0.02 & 0.04 & 0.06 & 0.12 \\
\hline & Treatment(T) & 0.005 & 0.01 & 0.01 & 0.03 & 0.01 & 0.01 & 0.02 & 0.03 \\
\hline & $\mathrm{G} \times \mathrm{T}$ & 0.025 & 0.05 & 0.07 & 0.14 & 0.03 & 0.06 & 0.08 & 0.17 \\
\hline
\end{tabular}

Means showing the same letters in a column do not differ significantly at a $5 \%$ probability level.

Data in parentheses indicate percentage increase (+) or decrease (-) over control 
Table.5 Stress response index (SRI) of 26 genotypes of groundnut under $200 \mathrm{mM} \mathrm{NaCl}$ for different growth parameters

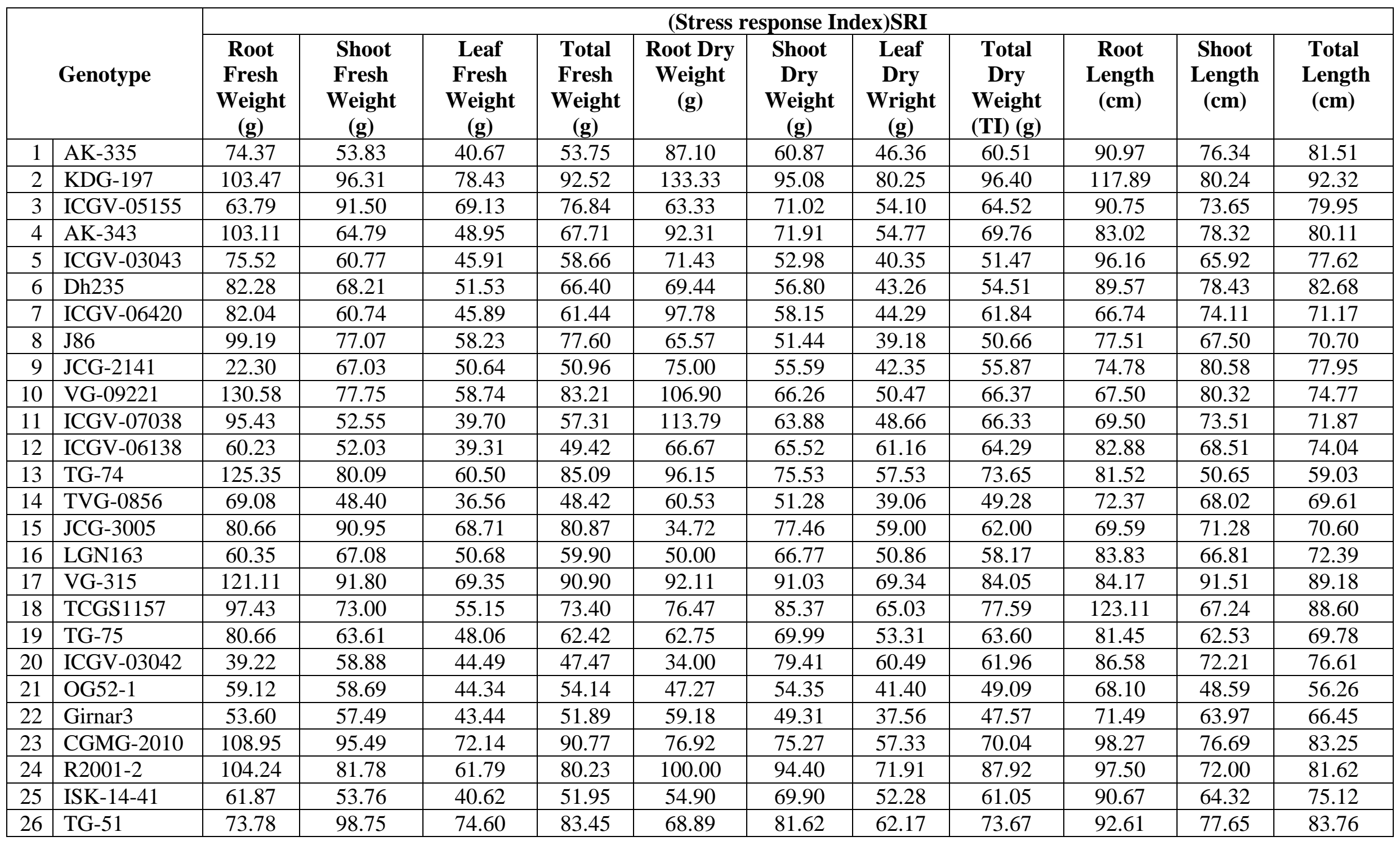


Table.6 Effect of salinity stress on total soluble sugar, proline, soluble protein and electrolyte leakage in tolerant and Susceptible genotypes

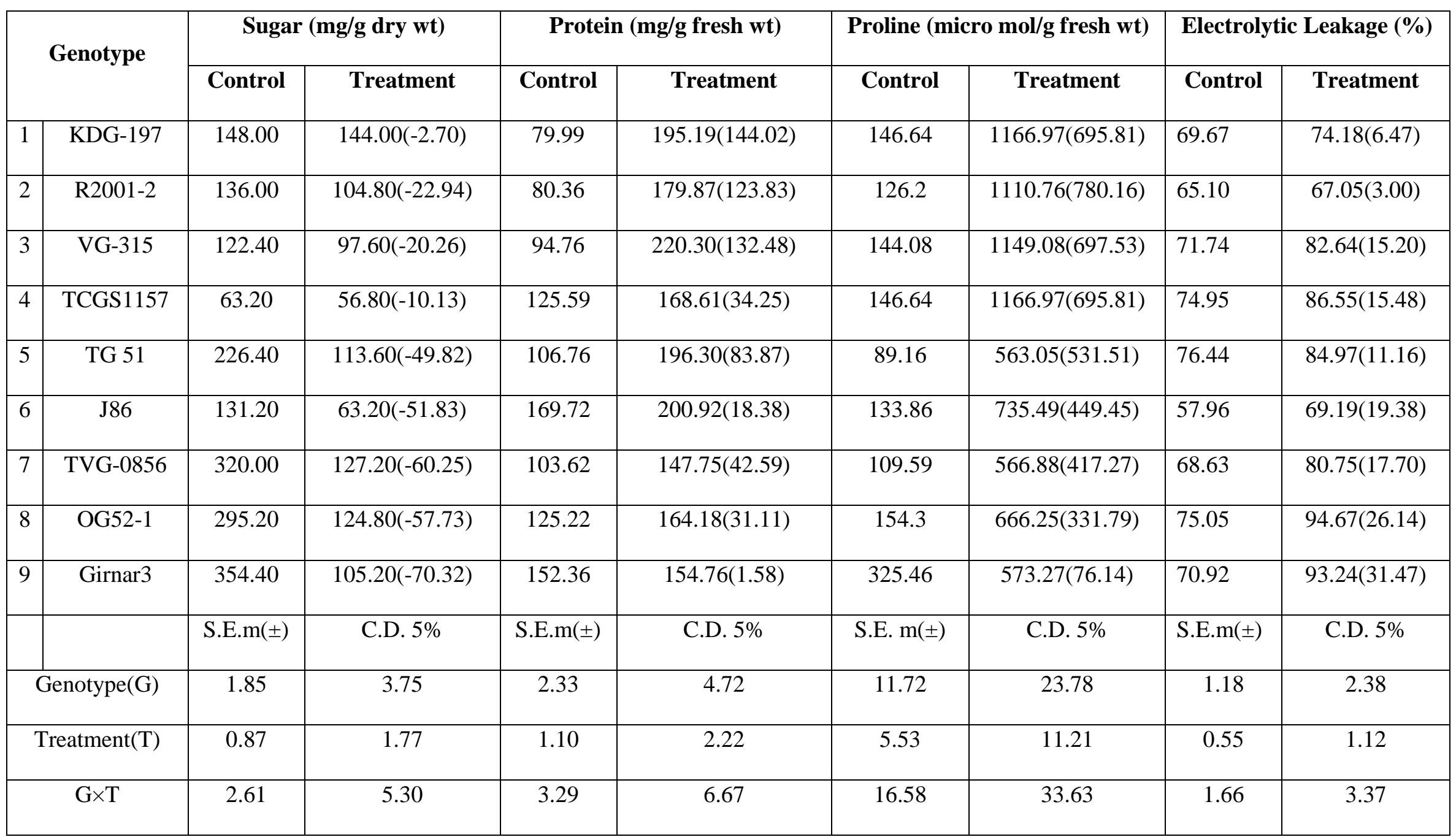

Means showing the same letters in a column do not differ significantly at a 5\% probability level.

Data in parentheses indicate percentage increase (+) or decrease (-) over control 
Fig.1 Dendrogram showing the clustering of 26 groundnut genotypes on the basis of salt tolerance

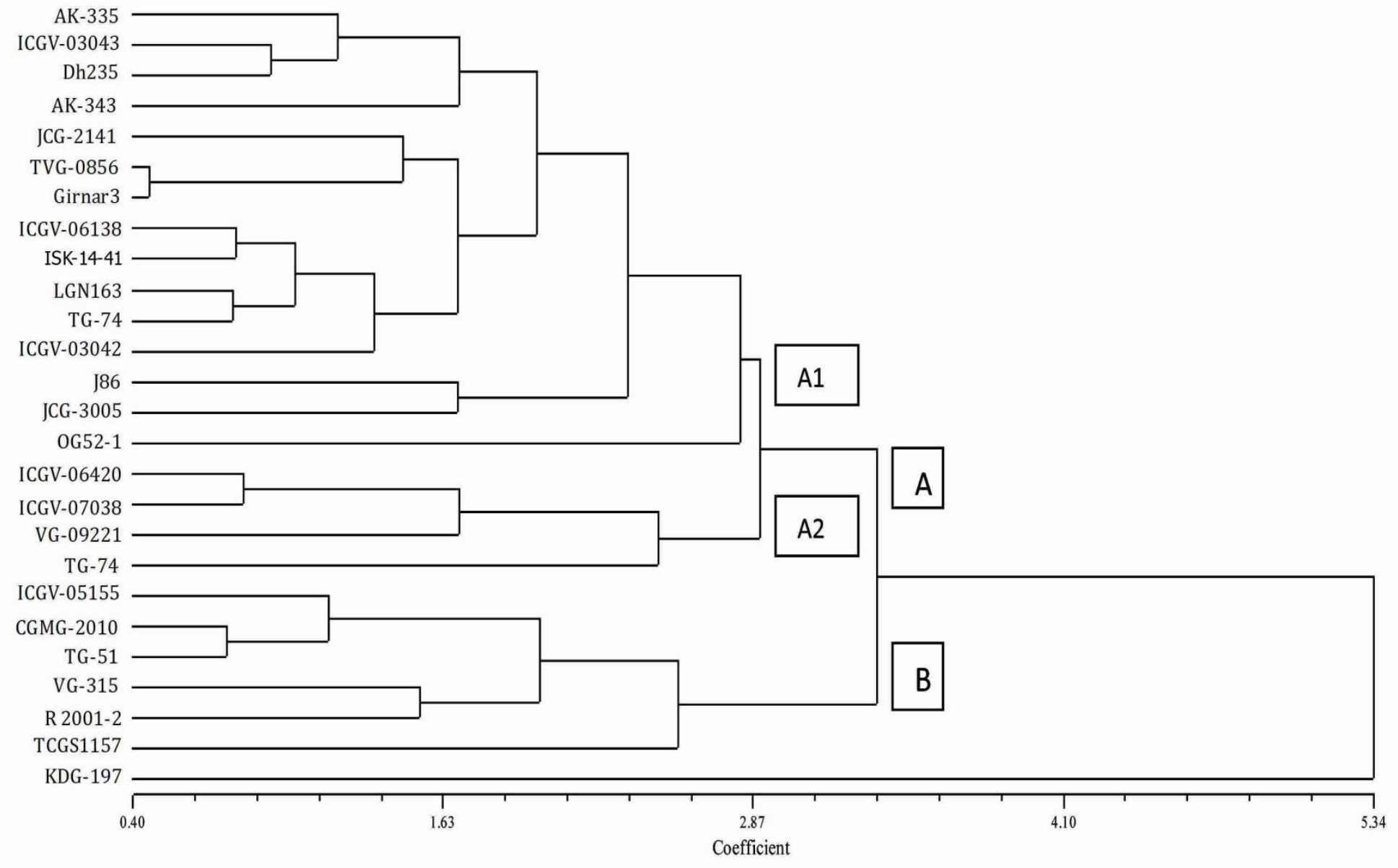


The salt tolerance relatively depends upon the intensity of salinity and relative performance of genotypes. Salt generally alters a wide array of metabolic processes culminating in stunted growth, reduced enzyme activities and photosynthetic carbon metabolism. The suppression of plant growth under salt-stress may either be due to osmotic reduction in water availability or to excessive accumulation of ions, known as specific ion effect. In this experiment five tolerant and four susceptible genotype showed remarkable difference in terms of different growth parameter and tolerance index under $200 \mathrm{mM}$ Salinity stress. The results are in agreement with those of Ghoulam et al2002 who reported that salinity caused a significant reduction in growth parameters of shoot and roots of sugarbeet. Sugars usually lower the osmotic potential of the cells favoring the holding of more bound water and reducing loss of turgidity. Therefore the accumulation of soluble sugars in the soluble pool suggests a protective role through osmoregulation (Singh and Rai 1983). In present study, reduction in sugar accumulation under salinity treatment might be attributed to inhibition of carbohydrate synthesis due to reduced rate of photosynthesis induced by salinity stress.

Tolerant genotype KDG-197 was the least affected among all the genotypes while susceptible genotype Girnar 3 was the most affected by salinity stress. Proline also induces the expression of salt-stressresponsive proteins and may improve the plant adaptation to salt-stress (Khedr et al 2003). The dramatic increase in proline content in the leaves under salinity stress was consistent with the role of proline as a compatible solute for osmotic adjustment during osmotic shock. However, in addition to its function as osmoregulator, proline might also play the role of osmoprotectant in stabilizing protein and scavenging. In this experiment higher accumulation of leaf proline in the salt tolerant genotypes might help them in osmotic adjustment in a better way as compared to the susceptible ones. this can be related to earlier findings in which Aazami et al (2010), found that an increase in proline content under salinity stress was probably due to the capacity of some plants to accumulate organic (sucrose, fructose and glucose) and inorganic $(\mathrm{Na}, \mathrm{K}$ and $\mathrm{Cl}$ ) metabolites in the cytoplasm to reduce the water potential and change the osmotic gradient, assuring the water flow to the plant and thereby might increase tolerance. The increase in leaf protein might be attributed to synthesis of some stress proteins induced by $\mathrm{NaCl}$ treatment. However, the tolerant and susceptible genotypes showed significant differences among them in respect of such increase. This results are consistent with Tort and Turkyilmaz (2004), Kapoor and Srivastava (2010). Electrolytic leakage is a valid, simple and quantitative indicator to the injury occurred to plasma membrane after exposure to salinity stress. The current study provided experimental evidence that the tolerant genotypes showed lesser extent of membrane damage as indicated by the change in EL\% under stress over control. Similarly, Lechno et al 1997 observed the same increasing trend of electrolyte leakage in salt sensitive cucumber cultivar as compared to the salt tolerant cultivar. This phenomenon was already observed by several authors in cucumber (Kaya et al 2001) and sugar beet (Ghoulam et al 2002).

The research revealed that KDG-197, R20012, VG-315, TCGS1157 and TG-51genotypes of Arachis hypogaea can withstand salt stress and produce good seedling vigour in sand culture with salinity up to $200 \mathrm{mM} \mathrm{NaCl}$, while other genotypes Girnar 3, OG52-1, TVG-0856 and J86 are highly susceptible to salt stress. Those that can withstand salt stress hold immense promise to be grown in the coastal saline areas, and can be used in 
breeding programmes for developing salttolerant variants of groundnut.

\section{Acknowledgement}

This work is supported by Scholarship (National Fellowship for OBC) awarded to Mr. Apurba Pal by University Grant commission, entrusted \& funded by Ministry of Social Justice \& Empowerment, Govt. of India, New Delhi. Authors are thankful to Department of Plant physiology, Faculty of Agriculture, Bidhan Chandra Krishi Viswavidyalaya, Mohanpur, Nadia, 741252, West Bengal.

\section{References}

Aazami, M. A., M. Torabi and E. Jalili 2010. In vitro response of promising tomato genotypes for tolerance to osmotic stress. Afr. J. Biotechnol., 9(26): 40144017.

Abogadallah, G.M., M. Serag and W.P. Quick. 2010. Fine and coarse regulation of reactive oxygen species in the salt tolerant mutants of barnyard grass and their wild type parents under salt stress. Physiol. Plant., 138: 60-73.

Bayuelo Jimenez J. S., Debouk, D. G. and Lynch J. P. 2002. Salinity tolerance in phaseolus species during early vegetative growth. Crop Si, 42:21842192

Chen, C., Tao, C., Peng, H. and Ding, Y. 2007. Genetic analysis of salt stress responses in Asparagus Bean (Vigna unguiculata (L.) ssp. sesquipedalis Verdc.). J. Hered., 98: 655- 665.

Chhabra, R., and Kamra, S. K. 2000. Management of salt affected soils. In: Extended Summaries, International Conference on Managing Natural Resources for Sustainable Agricultural Production in the 21st Century. Indian Soc of Soil Sci. 47-49.
Cokkizgin A., 2012. Salinity Stress in Common Bean (Phaseolus vulgaris L.) Seed Germination. Not Bot Horti Agrobo. 1(40):177-182.

FAO, 2005. http://www. faostat. org.

Ghoulam, C., Foursy, A. and Fares. K. 2002. Effects of salt stress on growth, inorganic ions and proline accumulation in relation to osmotic adjustment in five sugar beet cultivars. Environ. Exp. Bot., 47: 39-50.

Girdhar, I. K., Bhalodia, P. K., Misra, J. B., Girdhar, V. and Dayal, D. 2005. Performance of groundnut, Arachis hypogaea L. as influenced by soil salinity and saline water irrigation in black clay soils. Journal of Oilseeds Research, 22(1):183-187.

Guo, Z., Ou, W., Lu, S. and Zhong, Q. 2006. Differential responses of antioxidative system to chilling and drought in four rice cultivars differing in sensitivity. Plant Physiology and Biochemistry, 44: 828-836.

Kapoor, K., and Srivastava, A. 2010. Assessment of salinity tolerance of Vinga mungo var. Pu-19 using ex vitro and in vitro methods Asian J. Biotechnol., 2 (2) :73-85

Kaya, C., Kirnak, H. and Higgs, D. 2001. Effects of supplementary potassium and phosphorus on physiological development and mineral nutrition of cucumber and pepper cultivars grown at high salinity $(\mathrm{NaCl})$. J. Plant. Nutr, 24(9): 1457-1471.

Khedr, A. H. A., Abbas, M. A., Wahid, A. A. A., Quick, W.P. and Abogadallah, G. M. 2003. Proline induces the expression of salt-stress-responsive proteins and may improve the adaptation of Pancratium maritimum L. to salt-stress. $J$ Exp Bot 54:2553-2562

Lechno S., Zamski E., Telor E. 1997. Salt stress-induced responses in cucumber plants. J. Plant Physiol., 150: 206-211. 
Lowry, O. H., Rosebrogh, N. J., Farr, L. and Randall, R. J. 1951. Protein measurement with Folin phenol reagent. J.Biol.Chem, 193: 265- 275.

Mohanty, S.K., and Sridhar, R. 1982. Physiology of rice tungro virus disease: proline accumulations due to infection. Physiol. Plant., 56: 89- 93.

Murillo-Amador, B., Lopez-Aguilar, R., Kaya, C., Larrinaga-Mayoral, J. and Flores-Hernandez, A. 2002. Comparative effect of $\mathrm{NaCl}$ and $\mathrm{PEG}$ on germination emergence and seedling growth of cowpea. J. Agron. Crop Sci., 1(88):235-247.

Nithila, S., Durga, D. D., Velu, G., Amutha, R. and Rangaraju, G. 2013. Physiological Evaluation of Groundnut (Arachis hypogaea L.) Varieties for Salt Tolerance and Amelioration for Salt Stress. Research Journal of Agriculture and Forestry Sciences, 1(11):1-8.
Shannon, M. C., 1986. Breeding, selection and the genetics of salt tolerance. In: Salinity tolerance in Plants. (eds: Staples RC, and Toenniessn GH). John Wiley and Sons. 231-252.

Singh, G., and Rai, V. K. 1983. Response of two Cicer arietinum cultivars with differential sensitivities to water stress: Soluble Sugar and drought resistance. Indian J. Ecol., 10(2):11-15

Tort, N., and Turkyilmaz B. 2004. A physiological investigation on the mechanisms of salinity tolerance in some barley culture forms. J.F.S., 27:116

Yoshida, S., Forno, D. A., Cock J. H. and Gomoz, K. A. 1972. Laboratory Manual for Physiological Studies of Rice, 2nd edn. International Rice Research Institute, Loss Banos, Philippines.

\section{How to cite this article:}

Pal, A. and Pal, A.K. 2017. Physiological Basis of Salt Tolerance in Groundnut (Arachis hypogaea L.). Int.J.Curr.Microbiol.App.Sci. 6(9): 2157-2171. doi: https://doi.org/10.20546/ijcmas.2017.609.266 